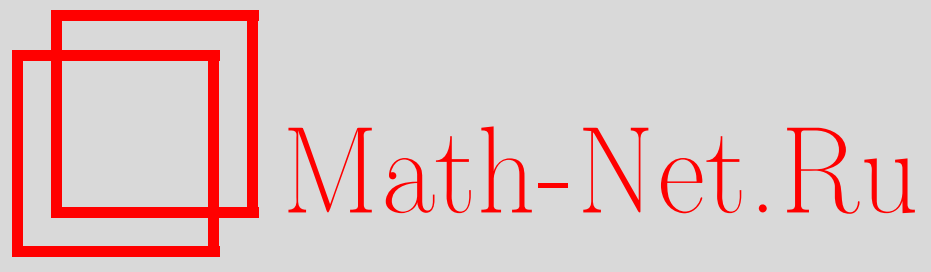

В. П. Маслов, Бозе-газ ангармонических осцилляторов и уточнение закона Ципфа, ТМФ, 2006, том 148, номер 3, 495-496

DOI: https://doi.org/10.4213/tmf2328

Использование Общероссийского математического портала Math-Net.Ru подразумевает, что вы прочитали и согласны с пользовательским соглашением http://www . mathnet.ru/rus/agreement

Параметры загрузки:

IP: 35.174 .16 .151

26 апреля 2023 г., 13:19:52

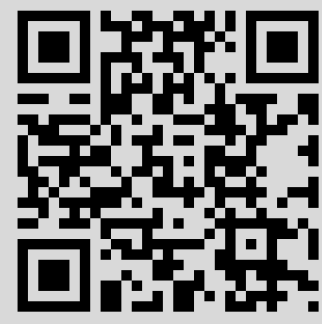




\section{БОЗЕ-ГАЗ АНГАРМОНИЧЕСКИХ ОСЦИЛЛЯТОРОВ И УТОЧНЕНИЕ ЗАКОНА ЦИПФА}

Приводится логарифмический закон, в пределе переходящий в закон Ципфа.

Ключевые слова: закон Ципфа, бозе-газ.

Будем нумеровать частицы в газе Бозе-Эйнштейна в любом порядке на данном уровне, начиная от нижнего уровня. Тогда номер первой частицы $N_{k}$ на уровне $\lambda_{k}$ равен числу частиц, лежащих на всех предыдущих уровнях энергии:

$$
N_{k}=\sum_{i=0}^{k} n_{i}=\sum_{i=0}^{k} \frac{1}{1-e^{-\beta \lambda_{i}+\sigma}} .
$$

Если $\lambda_{i}=i, k \gg 1, \beta \ll 1, \sigma \ll \beta$, то с точностью до $\beta^{3}$

$$
N_{k} \sim \sum_{i=1}^{k} \frac{1}{\beta i+\beta^{2} i^{2}} \sim \frac{1}{\beta} \int_{0}^{k} \frac{d x}{x+\beta x^{2}}=\frac{1}{\beta} \ln \left(\frac{\lambda_{k}}{1+\beta \lambda_{k}}\right) .
$$

Так как параметр $\beta$ мал, то эту формулу (см. (6) в работе [1]), вообще говоря, нельзя применить для больших $k$. Но если осциллятор ангармоничен [2], то эта формула справедлива и для больших $\lambda_{k}$ (при $\left.\lambda_{k}=k\right)$. Именно,

$$
N_{k}=\frac{1}{\beta} \ln \frac{\lambda_{k}}{1+\alpha \lambda_{k}},
$$

где $\alpha$ порядка единицы.

Пусть $N$ - число всех частиц, $\lambda_{s}$ - самый высокий уровень, на котором есть частицы, т.е. на уровнях $\lambda_{i}>\lambda_{s}$ частиц нет. Если частицы пронумеровать от частицы, находящейся на уровне $\lambda_{s}[3], \lambda_{s} \gg 1$, то номер частицы (ранг) $r$ будет равен $r_{k}=N-N_{k}$ - числу частиц на уровнях $\lambda_{i} \geqslant \lambda_{k}$. Вместе с тем

$$
N=\lim _{k \rightarrow \infty} \frac{1}{\beta} \ln \frac{\lambda_{k}}{1+\alpha \lambda_{k}}=\frac{1}{\beta} \ln \frac{1}{\alpha} .
$$

Следовательно,

$$
r_{k}=\frac{1}{\beta} \ln \left(1+\frac{1}{\alpha \lambda_{k}}\right)
$$

* Московский государственный университет, Москва, Россия. E-mail: v.p.maslov@mail.ru 
Отметим, что если мы будем пропускать все те уровни, на которых нет частиц, оставляя только уровни $\lambda_{k}^{\prime}$, на которых есть хотя бы одна частица, то формула (1) не изменится (ср. [3]). Тогда (1) будет соответствовать ранговому распределению типа распределения Ципфа.

В ранговых распределениях участвуют только целые числа - ранг (номер) $r$ объекта и число встречаемости $\omega_{r}$ объекта: задается ранг и ему сопоставляется число встречаемости. Если разность между ближайшими числами встречаемости $\omega_{l}$ и $\omega_{l+1}$ больше единицы, то говорят, что имеется лакуна с $\left(\omega_{l}-\omega_{l+1}-1\right)$ точками. Мы получаем ранговые распределения из обобщенного распределения типа Бозе-Эйнштейна для частиц [4], сопоставив частицам объекты более общего типа (например, слова в частотном словаре) и учитывая, что сумма всех частиц на уровнях $\lambda_{i}>\lambda_{l}$ есть ранг, отвечающий $\lambda_{l}$.

Это соотношение $\lambda_{i}=i+\gamma i^{2}$ (ангармоничность) получится, если мы сделаем дополнительное предположение относительно $N_{i}: N_{i+1}>N_{i}$ (предпочтение) в условиях теорем, приведенных в работах [4]-[6]. Полагая $\overline{\lambda_{i}}=\lambda_{i+1}-\lambda_{i}$, получим

а значит,

$$
\sum_{i=0}^{s} \overline{\lambda_{i}} N_{i} \equiv N_{s} \lambda_{s}-\sum_{i=0}^{s} n_{i} \lambda_{i}=\text { const }
$$

$$
\lambda_{j}=\sum_{i=0}^{j} \overline{\lambda_{i}}, \quad N_{k}=\sum_{i=0}^{k} \frac{\text { const }}{1-e^{-\beta \sum_{j=0}^{i} \overline{\lambda_{j}}}} .
$$

Поэтому, если $\overline{\lambda_{j}}=j$, то $\sum_{j=0}^{i} \overline{\lambda_{j}}=\left(i^{2}+i\right) / 2$.

В более общем случае аналогично предыдущему получаем [5], [6]

$$
r=\frac{T}{\alpha \gamma} \ln \left(1+\frac{1}{\alpha \omega^{\gamma}}\right)
$$

где $\omega$ - частота встречаемости объекта ранга $r$. При $\omega \rightarrow \infty$ имеется зависимость $r \sim$ $T /\left(\gamma \alpha \omega^{\gamma}\right)$ (закон Ципфа [7]). Значит, в случае, когда $T \gg 1$, параметр $\gamma$ может быть определен как $1 / D$, где $D$ - размерность, вычисленная из закона Ципфа, $T /(\gamma \alpha)-$ префактор [7]. Отсюда получаем

$$
\omega=\left(\frac{1}{e^{\alpha \gamma r / T}-1}\right)^{D} .
$$

Эта формула, как сказано в аннотации к книге [6], есть “квантовое продолжение законов Ципфа-Мандельброта и Парето для распределения капиталов".

\section{Список литературы}

[1] В. П.Маслов, Матем. заметки, 80:2 (2006), 220-230.

[2] Л. Д. Ландау, Е. М. Лифшиц, Квантовая механика, Физматгиз, М., 1963.

[3] В.П. Маслов, ТМФ, 147:3 (2006), 511-512.

[4] V.P. Maslov, Russ. J. Math. Phys., 12:4 (2005), 483-488.

[5] V.P. Maslov, Russ. J. Math. Phys., 13:3 (2006), 315-325.

[6] В. П. Маслов, Квантовая экономика, Наука, М., 2005.

[7] Б. Мандельброт, Фракталъная геометрия природы, Институт компьютерных исследований, М., 2002. 